\title{
Correlative Light and Electron Microscopy (CLEM) Techniques for Biological Samples
}

\author{
Vinod Nair $^{1}$, Forrest H Hoyt ${ }^{2}$, Thomas Hoenen ${ }^{3}$, Elizabeth R Fischer ${ }^{1}$
}

1. NIH/NIAID/ RML/RTB/EM Unit, 903 South $4^{\text {th }}$ Street, Hamilton, Montana 59840

2. Montana State University, Chemistry and Biochemistry, PO Box 173400 Bozeman, Montana 59717

${ }^{3 .}$ NIH/NIAID/ RML/Laboratory of Virology, 903 South $4^{\text {th }}$ Street, Hamilton, Montana 59840

Fluorescent light microscopy has been the technique of choice to visualize transient biological events or for performing quantitative microscopic analysis. Advanced super-resolution light microscopy techniques like STED have furthered these applications of fluorescent microscopy by overcoming the classical resolution limit [1]. However, this high-resolution information is limited to the interacting fluorescent molecules [2], thus leaving us with the lack of ultrastructural information from unlabeled regions of the cell. Correlative Light and Electron Microscopy (CLEM) overcomes this shortcoming by offering us the ability to not only quickly and accurately identify a cellular process by light microscopy but also obtain a higher resolution image of the same cell for structural analysis by electron microscopy. Nevertheless, special considerations have to be made while selecting fixatives, permeabilizing agents and solvents while processing a specimen to be imaged by both light and electron microscopy (EM). To this end, various reagents, solvents and resins were tested to develop a standardized processing technique that would enable favorable imaging using fluorescent light and electron microscopes. Additionally, studies were also carried out to determine ideal substrates that facilitate easy identification of cells in region of interest (ROI) in both light and EM investigations.

Figure 1 illustrates correlative light and transmission electron microscopy (TEM) study of Huh-7 cells infected with recombinant ebolavirus grown on a photo-etched alphanumerical coverslip (A). This pattern on the etched coverslip allowed for accurate identification of the ROI (B \& C). For imaging with light, cells were fixed in paraformaldehyde and imaged in phosphate buffered saline (PBS) using a laser confocal microscope. The samples were then fixed in $2.5 \%$ glutaraldehyde for better ultrastructural preservation and processed for TEM. Inclusions containing viral nucleocapsids (D \&E) were observed by TEM that correlate to mCherry labeled regions imaged earlier by fluorescent microscopy.

Figure 2 demonstrates correlative light and scanning electron microscopy (SEM) method. This technique involved the use of SEM finder grids to identify the cells of interest. Glass coverslips and formvar coated SEM finder grids were used as substrates to grow HeLa cells (A \&B) that were infected with $\mathrm{mCherry} \mathrm{labeled} \mathrm{salmonella}$ and imaged by light microscopy $(\mathrm{C})$. The specimens were then fixed with $2.5 \%$ glutaraldehyde and processed for SEM (D). As the formvar coated grids were too delicate to go through a critical point dryer (CPD), they were dried using Hexamethyldisilazane and later imaged using a SEM.

The techniques mentioned above can also be easily adapted to accommodate a range of Alexa Fluor ${ }^{\circledR}$ conjugated antibodies (data not shown). By growing cells in a $35 \mathrm{~mm}$ Petri dish imprinted with a relocation grid, this technique can be also extended to live cell studies. In comparison to CLEM techniques based on imaging semi thin sections; this technique enables investigators to collect the entire range of fluorescent signal through the 3 -axis volume. Additionally, completing the fluorescent imaging prior to EM processing permits use of all the reagents needed to preserve good ultrastructure without the 
concern of their effects on the fluorophores used. The strength of this CLEM technique lies in the ability to collect quality images using both light and EM.

\section{References:}

[1] S. W. Hell and J. Wichmann, Optics letters 19 (1994), p. 780-782.

[2] C. Loussert, C. L. Forestier and B. M. Humbel in "Methods in cell biology", Editor(s) T. MüllerReichert and P. Verkade, (Academic Press), 111 (2012), p. 59-73.

[3] The authors would like to acknowledge Dr. Leigh Knodler for the SEM samples and Anita Mora for assitance with graphic arts. This study was supported by Division of Intramural Research, NIAID, NIH.
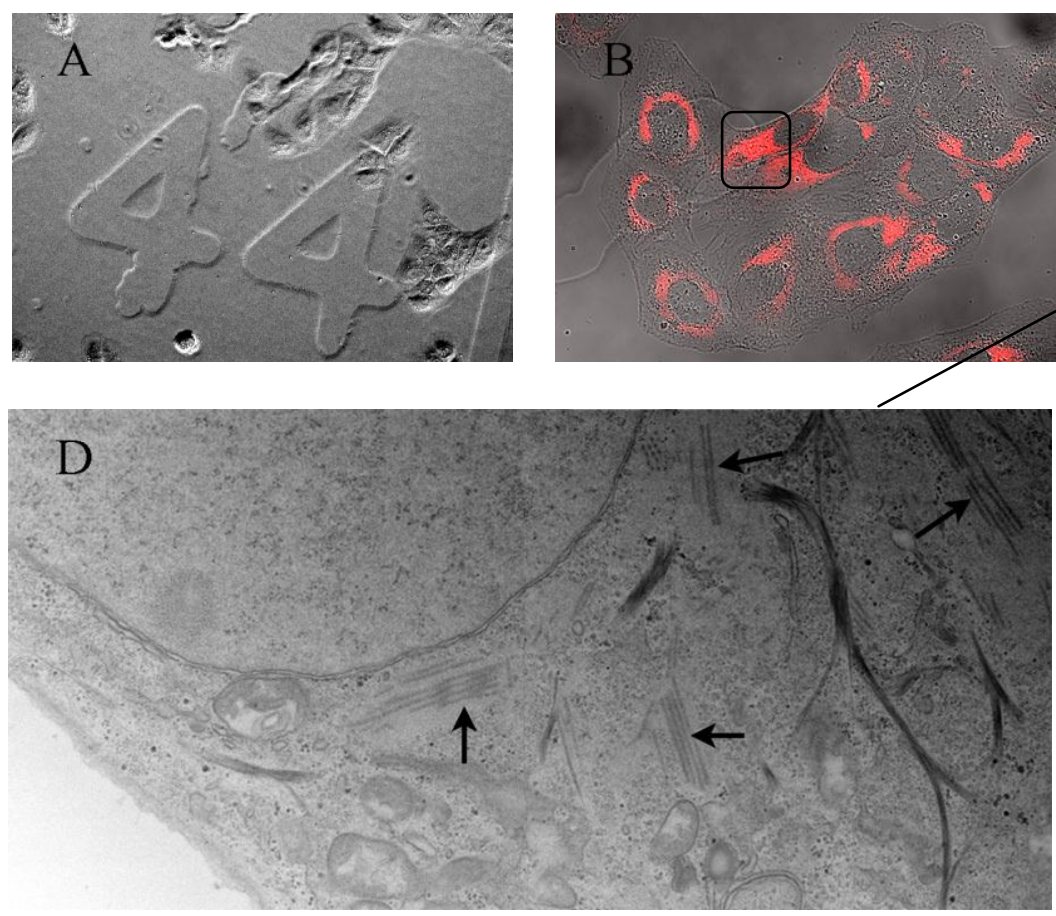
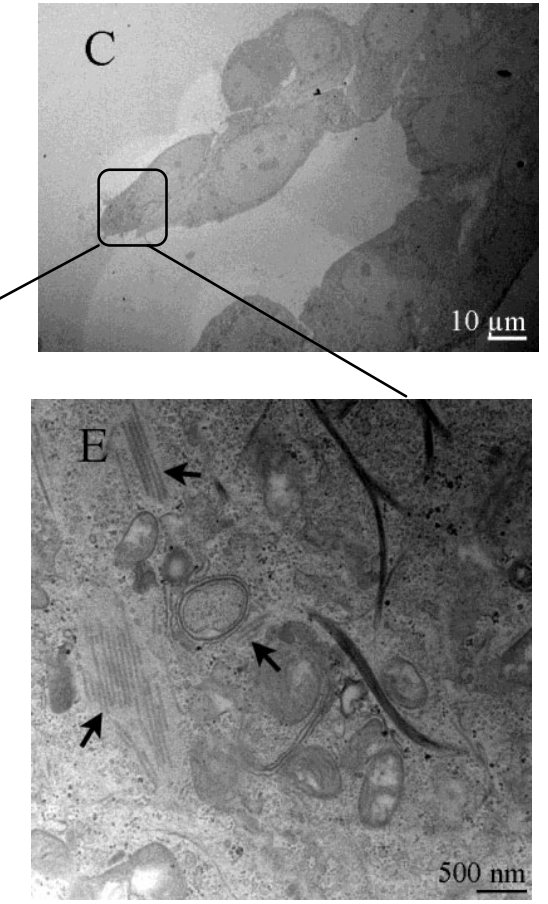

Figure 1. (A) HUH-7 cells infected with recombinant ebolavirus grown on a photo-etched glass coverslip. (B) Infected cells expressing mCherry viral polymerase. (C) Cell cluster located by TEM. (D \& E) High magnification images of the cell in ROI showing viral nucleocapsids (arrows)
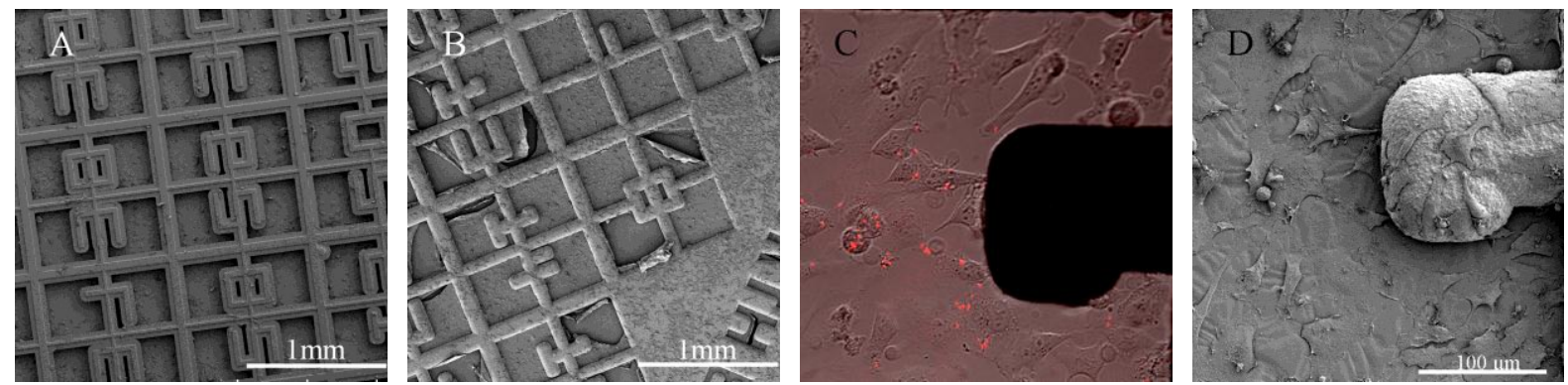

Figure 2. (A) HeLa cells grown on glass glued to SEM finder grid. (B) HeLa cells grown on formvar coated SEM finder grids. HeLa cells infected mCherry transformed salmonella were first imaged by confocal (C) and later imaged by SEM (D). 